Research Paper:

\title{
In-House Handmade Camera for Indocyanine Green Video Angiography
}

\author{
Amir Azarhomayoun ${ }^{1}$ (D), Mohsen Nouri2 ${ }^{*}$
}

1. Gundishapour Academy of Neuroscience, Ahvaz, Iran

2. Icahn School of Medicine, Mount Sinai, New York, USA

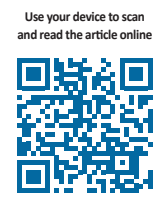

Citation Azarhomayoun A, Nouri M. In-House Handmade Camera for Indocyanine Green Video Angiography. Iran J Neurosurg. 2018; 4(1):13-18. http://dx.doi.org/10.32598/irjns.4.1.13

doif: http://dx.doi.org/10.32598/irjns.4.1.13

Funding: See Page 16

(c) Copyright: The Author(s)

Article info:

Received: 20 August 2017

Accepted: 20 November 2017

Available Online: 01 January 2018

Keywords:

Indocyanine green,

Angiography, Fluorescence

\begin{abstract}
Background and Aim: Vascular imaging during surgical procedures is very important and has many applications. There are several methods for intraoperative vascular assessment such as intraoperative angiography, Doppler, and fluorescence-based techniques. The latter group and specially the Indocyanine Green (ICG) Video Angiography (VA) is commonly used for vascular surgery and sentinel node biopsy. However, the cost of these microscope mounted cameras limit their availability in the developing countries. Considering this limitation, we designed and constructed a simplified low-cost camera for real-time ICG angiography. In this article, we describe the device structure and give a preliminary report on its usage in an animal model.
\end{abstract}

Methods and Materials/ Patients: ICG-VA camera was designed and constructed in our laboratory. The device consists of optic filters, light sources, and cameras.

Results: After inducing anesthesia and exposing mesenteric vessels in a mouse, ICG-VA of these vessels were taken and recorded.

Conclusion: Considering the very low cost of the device and its acceptable image quality, it can be utilized in operation theatres and or laboratories for research purposes.

\section{Introduction}

I

n operations with vascular preservation or reconstruction as the main goals, such as surgical treatment of brain vascular malformations, certainty of blood flow in vessels is of highest importance. Several methods are available for vascular patency assessment during the surgery, including intraoperative angiogra- phy, Doppler ultrasound, and Indocyanine Green (ICG) Video Angiography (VA) [1].

Intraoperative angiography is costly and its setup is not available in many operation theaters. Fluorescence techniques like ICG angiography are easily applicable, as they do not need moving the microscope out of the field and bringing the fluoroscopy in and have no radiation exposure. It helps surgeons with evaluating vascu-

* Corresponding Author:

Mohsen Nouri, MD

Address: Icahn School of Medicine, Mount Sinai, New York, USA

Tel: +98 (937) 5325455

E-mail:nouri@gan-ac.ir 
lar anatomy, integrity of blood vessels, and physiologic parameters of blood flow [2].

Nowadays microscopes are equipped with filters that allow ICG-VA during surgery. Unfortunately, these microscopes are very expensive and therefore, not available in many centers; especially the third-world countries. Considering these limitations, we constructed a handmade ICG-VA camera for angiography applicable in the operation room or the laboratory for research purposes. In this article, we reviewed the fluorescent properties of ICG briefly and then, described the structure of our device and its preliminary usage in an animal model.

\section{Methods and Materials/Patients}

\section{ICG properties and camera design}

ICG is a fluorescent substance with an excitation wavelength between 600 to $900 \mathrm{~nm}$ (peak of absorption 800 $\mathrm{nm}$ ) in plasma and fluorescence wavelength between 750 to $900 \mathrm{~nm}$ (peak $830 \mathrm{~nm}$ ). Fluorescence happens only if the ICG is bound to proteins (e.g. in blood). Our camera was designed and constructed, based on these properties (Figure 1).

This device is composed of four light sources and 2 cameras (Figure 2). One light source provides pure yellow light $(560 \mathrm{~nm})$ to the field for recording the surgical field by a regular camera. Ceiling light cannot be used for this purpose, as most light sources do not provide pure rays and also emit some light near the infrared range (wavelength above $800 \mathrm{~nm}$ ) which interferes with fluorescence angiography recorded by the other camera. But, as explained below, pure yellow light gets filtered by the ICG camera thus preventing any image artifacts.

The other light source is a projector of $775 \mathrm{~nm}$ wavelength (it has a range of 750 to 830 with a 775 peak) with a short-pass filter of $780 \mathrm{~nm}$ in front of it to prevent any ray with wavelength above $780 \mathrm{~nm}$ from reaching the field. Charged Couple Device (CCD) surveillance cameras (Sony Super HAD II CCD image sensor with 650 TVL resolution, assembled in Iran) were used for recording. One camera is for recording of the surgical field under the yellow light.

The other camera is to record ICG-VA and has a longpass $830 \mathrm{~nm}$ filter (Marumi, Japan) to filter out lights below $850 \mathrm{~nm}$ wavelength. Figure 2 shows the device components. Both cameras were connected to a 4- channel Digital Video Recorder (DVR) for simultaneous recording of the videos. The third light source constitutes of four parallel $780 \mathrm{~nm}$ lasers to illuminate the center of surgical field. In our experience, this setting results in a sharper image contrast.

Normally, the ICG camera shows a dark image as all visible light (below $830 \mathrm{~nm}$ ) are filtered; so even the near-infrared light $(780 \mathrm{~nm})$ by the second and third projectors is not detected. Therefore, it is impossible to understand if the camera lens is focused on the object.

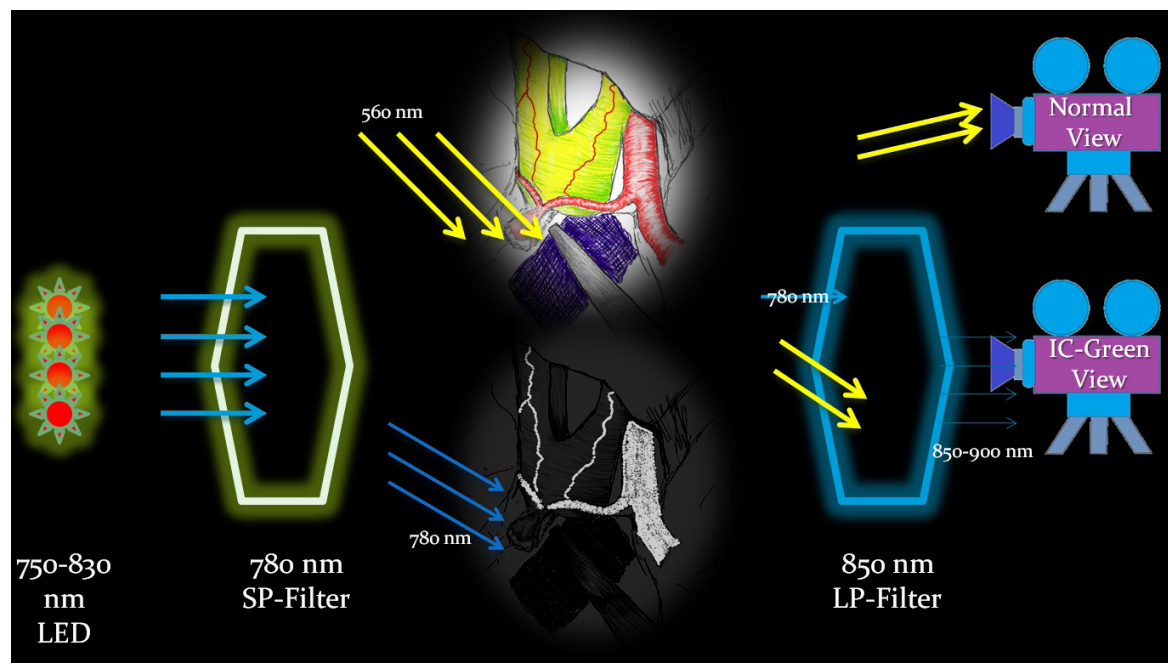

Figure 1. Biophysics of the ICG and the designed camera

The surgical field is being exposed to yellow $(560 \mathrm{~nm})$ and near-infrared (NIR, $780 \mathrm{~nm}$ ) lights. The reflected light from the vessels containing indocyanine green is within the range of 750 to $900 \mathrm{~nm}$. By using an 830 to $850 \mathrm{~nm}$ long pass filter in front of the camera, only 850 to 900 $\mathrm{nm}$ wavelengths are allowed to reach the camera. Lights from the yellow and NIR projectors are blocked by this filer. Therefore, the entire field will be dark except for the fluorescence reflecting vessels. The yellow filter illuminating the surgical field is reflected and detected by the regular camera to record the surgery. 


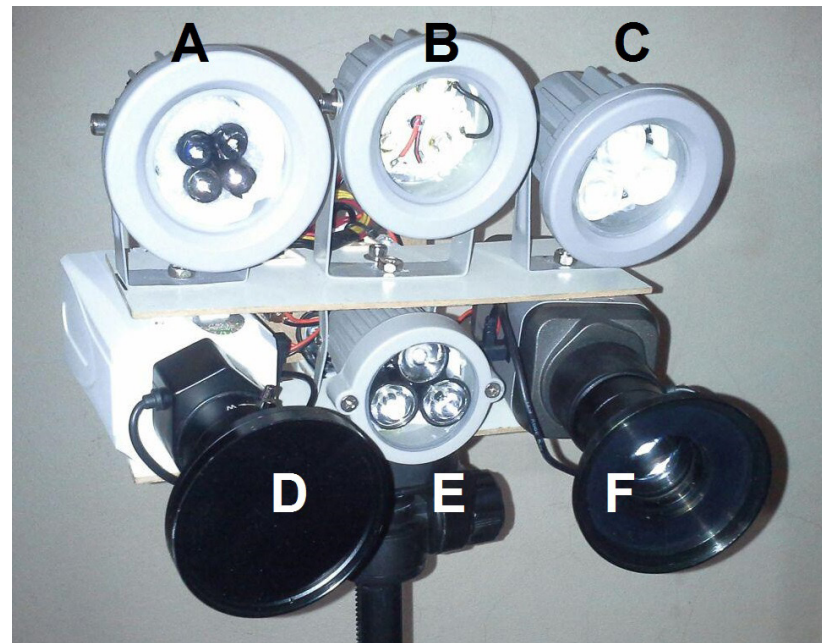

Figure 2. In-house handmade indocyanine green angiography camera

A. Four parallel $780 \mathrm{~nm}$ lasers; B. light emitting diodes at $775 \mathrm{~nm}$ with a short pass $780 \mathrm{~nm}$ filter; C. yellow light emitting diodes; D. ICG camera with long pass $830 \mathrm{~nm}$ filter; E. Light emitting diodes at $950 \mathrm{~nm}$ wavelength to adjust and focus the filtered camera; F. Regular camera without filters to record normal surgical field

To solve this problem, the fourth light source was added to emit infrared light $(950 \mathrm{~nm})$ which is not filtered by the ICG camera and illuminates the field while we can focus the ICG camera lens. After adjusting the lens, this projector is turned off.

\section{Animal models}

Helsinki Declaration for animal care was followed throughout the study in all experiments performed. Balb/c female mice (20-25 g) were purchased form the
Faculty of Veterinary Medicine, University of Tehran, Iran. Ketamine 50 mg/kg (Alfasan, Woerden, The Netherlands) and acepromazine $5 \mathrm{mg} / \mathrm{kg}$ (Castran, Interchemie, The Netherlands) were used to induce anesthesia for laparotomy.

To have a good source of vessels, mesenteric arteries of the small intestine were exposed. Animal's tail was used to inject ICG intravenously with an insulin syringe (1 $\mathrm{mg} / \mathrm{kg}$ ). Intraperitoneal injection of sodium pento-

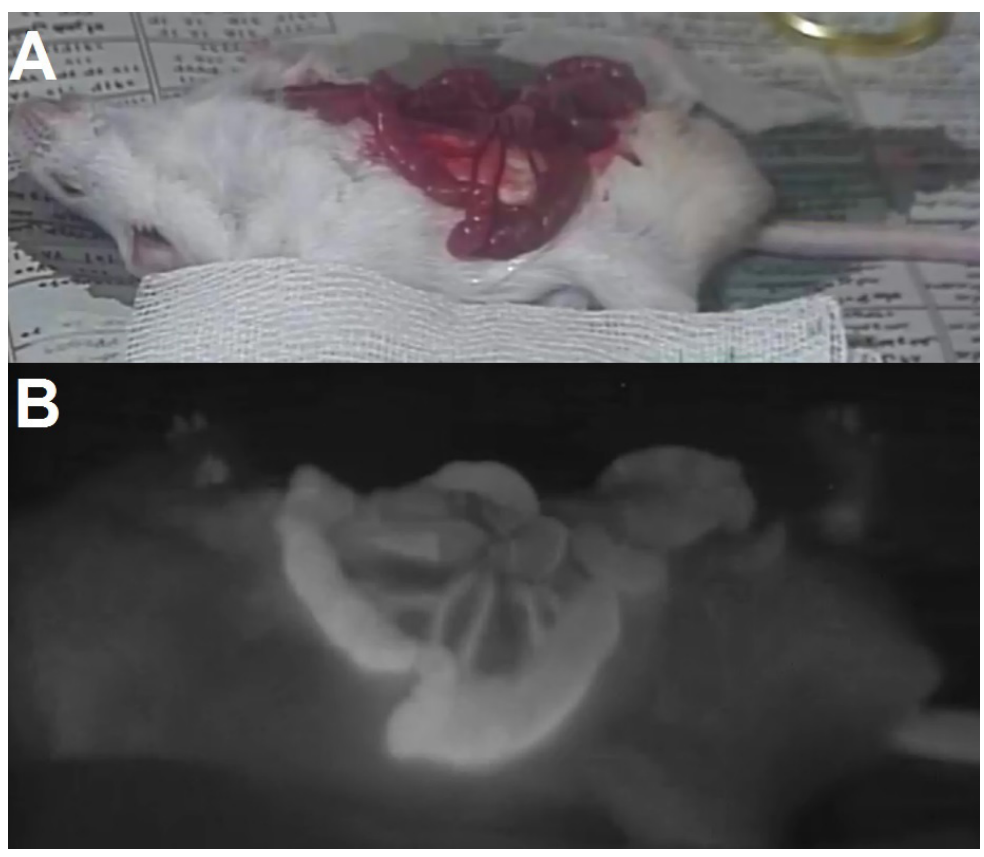

Figure 3. Simultaneous recording of the regular surgical field (A) and indocyanine angiography of the exposed mesenteric arteries (B) 
barbital (120 mg/kg) (Sigma, MO, USA) was given to euthanize the animal after the procedure.

\section{Results}

After inducing anesthesia and exposing mesenteric vessels (as mentioned earlier), projector and laser were turned on to illuminate the surgical field. Indocyanine green (SERB, Paris, France) was reconstituted with sterile water for intravenous injections (1 mg/ $\mathrm{kg})$. We introduced ICG through central vein of mouse tail after darkening the room. The ICG in the vessels was exposed to the $780 \mathrm{~nm}$ light and its fluorescent light was detected by the filtered Camera Connected to DVR and TV (CCTV setup). Simultaneous recording of the surgical field was done by the regular camera under the yellow light (Figure 3).

\section{Discussion}

There are some reports available of handmade devices for fluorescence applications [3, 4]. Fujisawa et al. constructed an ICG imaging system for sentinel node detection by ICG and successfully used it in 16 patients [3]. Pallota et al. manufactured a custom-made system for ICG detection of lymphatic vessels in flap reconstructed lower extremities to predict lymphedema in these patients [4]. Szyc et al. designed a handhold ICG fluorescence imaging system for sentinel node biopsy [5].

None of the above-mentioned devices were applied for ICG arterial angiography and only used for lymphangiography which does not require high quality imaging and contrast. In our experiment, we tried a stepwise manner to improve the quality of images by using more near-infrared sensitive cameras and also, strengthening the power of illuminating projectors.

ICG angiography is applicable in the operation rooms or laboratories [2]. Intraoperative ICG angiography has been reported to correlate well with the findings of intra- or post-operative conventional angiography [6]. However, this costly technology may not be affordable in many developing countries and the use of low-cost hand-made devices may help in these situations to reduce the cost while maintaining the clinical outcome [7].

Our camera was designed with some limitations. Due to the international sanctions against the country, we lacked accessing high quality products in many instances. For example, using medical grade cameras specifically designed for near-infrared wavelength, instead of CCDs improves the quality of the images significantly.
High power emitting diodes and lasers can also improve the contrast and sharpness of the images which were inaccessible for us.

Also, due to the regulations by the Ministry of Health and Medical Educations, we had extremely limited access to the ICG drug which prevented repetition of our experiment. Therefore, we believe that researchers in other developing countries with a wider access to electronic and optic resources may achieve higher quality images with few modifications in our proposed camera. Currently, we are using the camera in our laboratory to develop a blood flow analysis software comparable to those available in the market [2]. We are also working to create the first generation of real-time stereoscopic ICG angiographies (unpublished data).

\section{Conclusion}

Considering the very low cost of the device and its acceptable image quality, it can be utilized in operation theatres and or laboratories for research purposes.

\section{Ethical Considerations}

\section{Compliance with ethical guidelines}

There was no ethical principles to be considered in this research.

Funding

The project was financially supported by Jundishapur Academy of Neuroscience and no external fund was received for any part of the study.

\section{Conflict of interest}

All authors certify that they have no affiliations with or involvement in any organization or entity with any financial interest (such as honoraria; educational grants; participation in speakers' bureaus; membership, employment, consultancies, stock ownership, or other equity interest; and expert testimony or patent-licensing arrangements), or non-financial interest.

\section{Acknowledgements}

The authors extend their gratitude to Professor Yoko Kato from Japan for her dedication to educate young neurosurgeons from developing countries which resulted in our familiarity with intraoperative angiography and development of this project. 


\section{References}

[1] Yamada Y, Kato Y, Nouri M. No overtaking! Take a safe trip to aneurysm. Austin Journal of Cerebrovascular Disease \& Stroke. 2014 $1(2): 1$.

[2] Kato Y, Yamada Y, Sadato A, Nouri M, Cherian I, Tanaka T, Inamasu J. Intraoperative anatomical and hemodynamic analysis of intracerebral arteriovenous malformations by semi-quantitative color-coded indocyanine green videoangiography. Asian Journal of Neurosurgery. 2017; 12(4):638-43. [DOI:10.4103/ajns.AJNS_62_14] [PMID] [PMCID]

[3] Fujisawa Y, Nakamura Y, Kawachi Y, Otsuka F. A custom-made, lowcost intraoperative fluorescence navigation system with indocyanine green for sentinel lymph node biopsy in skin cancer. Dermatology. 2011; 222(3):261-8. [DOI: 10.1159/000327080] [PMID]

[4] Pallotta OJ, van Zanten M, McEwen M, Burrow L, Beesley J, Piller N Development and validation of a custom made indocyanine green fluorescence lymphatic vessel imager. Journal of Biomedical Optics. 2015; 20(6):066003. [DOI:10.1117/1.JBO.20.6.066003] [PMID]

[5] Szyc L, Bonifer S, Walter A, Jagemann U, Grosenick D, Macdonald R. Development of a handheld fluorescence imaging camera for intraoperative sentinel lymph node mapping. Journal of Biomedical Optics. 2015; 20(5):051025 [DOI:10.1117/1.JBO.20.5.051025] [PMID]

[6] Raabe A, Nakaji P, Beck J, Kim LJ, Hsu FP, Kamerman JD, et al. Prospective evaluation of surgical microscope-integrated intraoperative near-infrared indocyanine green video angiography during aneurysm surgery. Journal of Neurosurgery. 2005; 103(6):982-9. [DOI:10.3171/jns.2005.103.6.0982] [PMID]

[7] Kato $\mathrm{Y}$, Nouri M. Training the next generation of neurosurgeons in developing countries: Mission possible. Iranian Journal of Neurosurgery. 2017; 3(1):6-7. [DOI:10.29252/irjns.3.1.6] 
